La obra incorpora distintos niveles de análisis, evidencia de distintos países, tablas resumen, estudios de caso, propuesta pedagógica y una muy abundante bibliografía, Hubiera resultado muy útil incluir una robusta conclusión que cerrara la discusión, aludiendo, primero, a que el análisis se hace en distintos planos; segundo, que hay propuestas que apuntan hacia recomendaciones y, tercero, que aparece en el texto un esfuerzo didáctico que se desdobla, incluso, en forma de lecciones. Es un libro que aborda un tema muy relevante, escrito por una de las más reconocidas especialistas, que logra transmitir no sólo su entusiasmo por lo mucho que ha hecho y escrito en este campo, sino incluso su pasión.

\title{
María José Canel, La comunicación de la administración pública. Para gobernar con la sociedad, México, Fondo de Cultura Económica, 2018, 542 pp.
}

Por Grisel Salazar Rebolledo, CIDE

La comunicación no es un acto voluntario, es un elemento inherente al ser humano y a su interacción con los otros. El ámbito público no es la excepción. En él, todo comunica: desde los hechos más deliberados de transmisión de mensaje, como las conferencias de prensa o las fotografías de inauguraciones o atención a zonas de desastre, hasta los sucesos más circunstanciales, como el trato, malo o bueno, que se otorga en una oficina de gobierno, o los más inesperados, como la entrevista improvisada que se organiza en la banqueta de un edificio de gobierno. En la administración pública, como esa primera ventana de contacto ciudadano, la comunicación es fundamental como elemento de legitimidad y de gobernabilidad, y es un elemento al que ningún funcionario puede renunciar. Paradójicamente, hasta la fecha no se había dedicado ningún libro al estudio de la comunicación en la administración pública, ni se había ofrecido una delimitación conceptual ni una guía para su ejercicio.

La comunicación en el ámbito público ha estado centrada tradicionalmente en la política, en las campañas electorales, en la construcción de la imagen pública; temas que han generado cúmulos de libros teóricos, incluyendo obras de la propia Canel, manuales prácticos y ejercicios de consultoría. Sin embargo, justo esa parte de la comunicación gubernamental es la más contingente y también la más efímera. Lo permanente es la comunicación de la administración, que es en última instancia la política en acción. Es llamativa la ausencia de estudios dedicados a analizar cómo transmitir mejor a los ciudadanos la forma en la que 
se formulan o se evalúan las políticas públicas. Más allá de las figuras decorativas de un buzón de quejas, un portal de Internet o una cuenta de Twitter obligatoria para todos los funcionarios, ¿cómo se abre un verdadero canal de comunicación efectiva con la ciudadanía? y dado que la comunicación transcurre de manera permanente, ¿cómo pueden los administradores públicos estar alertas y equiparse para comunicar mejor? Canel acepta el desafío de responder estas preguntas que, por diversas razones, la literatura había dejado de lado.

A diferencia de la comunicación en campañas políticas, que tiene un objetivo muy claro - ganar elecciones - la estrategia comunicacional de la administración se enfrenta al reto de reconstruir o conservar la confianza del ciudadano. Es una tarea de más largo aliento, que exige demostrar una serie de virtudes del buen gobierno más allá de la vehemencia de las campańas electorales, pero en correspondencia con la coyuntura del momento. La comunicación de la administración pública, según se desprende del libro de Canel, no sólo supone una transmisión de propuestas, sino también una gestión efectiva de las expectativas que se generan con ellas (p. 267).

La preservación de la legitimidad que se ganó en las urnas en mucho depende de la buena estructuración de un mensaje, señala Canel, recordando en mucho lo que ya señalaba Giandomenico Majone: quien gana el argumento, gana la política. Gobernar, nos dice Majone, es argumentar la selección de una decisión pública por encima de otras. Dado que no existen causas impecables, visiblemente objetivas, entre los problemas públicos y sus soluciones, la persuasión es un arte que el gobierno debe cultivar casi por principio.

María José Canel, reconocida estudiosa de la comunicación pública, oportunamente se propone contribuir a llenar este nicho con su libro La comunicación de la administración pública. Para gobernar con la sociedad, en el que retoma preocupaciones clásicas de la administración pública, como la recuperación de la confianza ciudadana y la construcción de gobiernos eficientes, pero también al introducir desafíos de vanguardia, como los que imponen las nuevas tecnologías y aquello que se ha llamado la "posverdad".

El libro responde a una preocupación central: existe una brecha entre lo que el gobierno hace, lo que la administración dice que hace y lo que el ciudadano percibe que se hace. Esta brecha no es sino el reflejo de una estrategia de comunicación deficiente, que provoca frustraciones tanto en la administración, que es incapaz de transmitir su acción, como en el público, que percibe ineficiencia, indiferencia o incapacidad. Por eso, de acuerdo con Canel, la comunicación efectiva de una decisión pública equivale a dotarla de un valor agregado. Una 
buena estrategia de comunicación es, en esa medida, la creación de un bien público intangible.

De forma pertinente, Canel inicia la discusión con una delimitación conceptual necesaria de la comunicación de la administración pública, precisamente para diferenciarla de la comunicación política gubernamental o de las campañas electorales. La comunicación de la administración pública se refiere al:

intercambio de realidades cognoscitivas, que se realiza entre las organizaciones públicas, y entre éstas y los públicos clave, orientado a asistir en el cumplimiento de la finalidad (la búsqueda del bien común), a través de los servicios públicos, encarnado en una estructura orgánica de dimensión territorial y que articula y delimita la legitimidad del poder (p. 40).

En ese sentido, el concepto hace referencia a dos ideas principales, primero, es una transmisión de ida y vuelta (y no un mensaje unilateral) y segundo, ocurre en una red de actores que incluye las interacciones entre las propias organizaciones de gobierno. Estos aspectos raramente se consideran en la elaboración de las políticas públicas, a pesar de que en ellos residen con frecuencia cuellos de botella que terminan por deslegitimar hasta la política mejor construida.

La comunicación de la administración pública es una obra profusa y extensa, que da cuenta de la complejidad del entorno que enfrenta el mensaje de la administración, no sólo desde una perspectiva teórica, sino con una marcada vocación práctica, pensando en constituirse en un manual de consulta constante para los administradores, con ejercicios prácticos que invitan a la reflexión sobre las pautas comunicativas, intencionales o no, de las instituciones públicas y al diseño o rediseño de las estrategias comunicacionales.

El libro está organizado en torno a seis partes: "Marco introductorio", "Los problemas de partida: desconfianza, posverdad y politización", "La comunicación de la administración pública y la red", "Escuchar para gobernar con la sociedad", "Necesitamos organizarnos mejor" y "Evaluar para retroalimentar". Estos seis capítulos están atravesados por dos temas sustanciales: el ciudadano como la razón de ser de la comunicación de la administración pública y los medios de comunicación como ese entorno en el que se mueven los mensajes.

En primer lugar, colocar a la ciudadanía como centro del análisis es un elemento de gran acierto en un momento en el que los paradigmas de la administración pública actual han colocado fuera de los reflectores a los actores públicos como únicos responsables de la resolución de los problemas colectivos. Hace tiempo ya que la ciudadanía ha dejado de ser vista como menor de edad, que ha demandado nuevas formas de gobernar — con menos jerarquías y más apertura; 
con menos imposición y más capacidad de respuesta-. Estas nuevas formas de administrar los asuntos públicos también necesitan una nueva forma de comunicar, en un momento en el que la rendición de cuentas es permanente, la discusión de las decisiones públicas y de sus resultados en foros y redes sociales es continua y ya no es asunto exclusivo de especialistas. Mucho menos corresponde a los gobiernos indicar cómo debe entenderse una política pública ni predicar sobre su éxito o fracaso; al menos no sin ofrecer evidencia sobre esa valoración. Por esa razón, resulta muy pertinente que la preposición elegida por Canel en el subtítulo de su libro sea comunicar "con" la sociedad y no "para" la sociedad, pues eso remitiría a una forma de condescendencia que corresponde a modelos jerárquicos, pero no a una visión de corresponsabilidad. Al incluir a la sociedad en la acción comunicativa, Canel alude a una verdadera "gobernanza" del mensaje, en el que ambas partes intervienen y ambas son corresponsables.

Un acierto de Canel es que el desarrollo de las habilidades comunicacionales no sólo se refiere a una estrategia retórica, sino a una coherencia entre dichos y hechos. No se trata de comunicar exclusivamente buenas noticias o logros de gobierno, sino de saber transmitir con talento la forma en que se han enfrentado coyunturas complejas, incluso si eso implica reconocer honestamente fallos de política. Esto es de suma relevancia en un momento en que, como nunca antes, la plaza pública se ha vuelto vibrante, visible y abierta a discutir, a juzgar los detalles de las decisiones públicas, y también a intervenir directamente en ellas. Los ejercicios de monitoreo y evaluación de políticas emprendidos desde la sociedad civil (y que muchas veces se realiza con información que el propio gobierno provee) se insertan en esta capa adicional de desafíos para la administración, en tanto pueden entrar en conflicto como mensajes contradictorios o como interlocutores con legitimidad en disputa. Ningún plan de comunicación actual puede omitir que las políticas constituyen una argumentación bilateral y no un monólogo. Y es que Canel no es ingenua. Desde el primer momento señala como uno de los problemas de partida que "por default, los ciudadanos desconfían de los administradores" (p. 101), así, hay todo un camino por recorrer para recobrar la confianza, que depende, en gran medida, del talento para pasar de la cultura del control a la cultura del compromiso con el ciudadano, y de la eficacia para comunicar mensajes congruentes con este compromiso.

El segundo eje conductor del libro se refiere a los medios de transmisión de los mensajes, y aunque el énfasis puesto por Canel está en las redes sociales, los medios tradicionales de comunicación no pueden obviarse en esta discusión. La transmisión del mensaje no ocurre en el vacío y por esa razón es indispensable la reflexión 
sobre el papel que desempeñan los medios en la estructuración de las campañas de comunicación de la administración pública. Los medios constituyen filtros que se interponen entre lo que la administración busca comunicar y lo que el ciudadano percibe, y pueden constituirse en sustitutos o complementos de dichas estrategias de comunicación, al proveer elementos para la evaluación de las decisiones públicas y al constituir ellos mismos una voz que puede estar revestida de mayor legitimidad o mayor eficacia para comunicar un mensaje que la propia administración.

Los medios de comunicación, como traductores y proveedores de sentido de la acción pública para las audiencias, pueden convertirse en el mejor aliado de la administración o en uno de los mayores obstáculos para la comunicación. En cualquiera de los dos casos, son una voz que la administración no puede pasar por alto, especialmente frente a prácticas novedosas que transcurren en medios y redes sociales, como la verificación en tiempo real de los dichos de los funcionarios públicos o los repositorios de información sobre políticas públicas. Las herramientas de transparencia, que se han vuelto insumos básicos para el periodismo de investigación, también constituyen, a decir de Canel, uno de los más importantes desafíos que enfrenta el mensaje de la administración pública, que tiene que lidiar con el contraste continuo (y democráticamente muy deseable) que llevan a cabo los medios de comunicación independientes. Así, una advertencia que, aunque parezca obvia, rara vez es considerada por administradores y funcionarios es que, dado que todo acto comunica, es indispensable estar alerta a las incongruencias que puedan existir entre el mensaje planeado y el comportamiento no planeado, pues en general los focos mediáticos recaerán sobre el conflicto y la contradicción.

Además de estos elementos, María José Canel resalta fenómenos recientes directamente desprendidos de la evolución de las tecnologías de la información, como la proliferación de datos, o big data, y la forma en que pueden ayudar a mejorar la relación entre administración y ciudadano (p. 281), o los retos de la posverdad, como elemento que "banaliza contenidos" y "genera desconfianza en las instituciones" (p. 124).

Así, puede verse que, aunque el libro de Canel es uno que se cuenta desde la administración pública y con un perfil muy práctico, orientado a los propios administradores, en realidad es una obra que tiene gran interés para todos aquellos profesionales y estudiosos de la comunicación que tengan inquietud por entender cómo se estructura, cómo se recibe y cómo se puede distorsionar el mensaje sobre las acciones públicas. Los estudiosos de la evolución de la administración pública también encontrarán en la lectura de esta obra ideas muy sugerentes para analizar 
la manera en la que la gobernanza también debe trasladarse a la comunicación de las decisiones públicas.

Además, para el lector mexicano es una obra que tiene interés especial a la luz de la discusión actual sobre los excesivos gastos de una política de comunicación que se siente ineficiente y alejada de su objetivo original. Las diversas reflexiones a las que invita Canel a lo largo de sus capítulos sin duda pueden proveer elementos originales para la articulación de una mejor política de comunicación social.

Uno de los mensajes centrales del libro de Canel es que la comunicación es un mensaje de ida y vuelta. Hoy, en las antípodas del modelo comunicacional de prédica política, el libro de Canel resulta especialmente pertinente para considerar si, más allá de los ejercicios de apertura de canales y de mejores campañas comunicacionales, los gobiernos realmente están preparados para escuchar. Y si lo están, una vez que escuchen, ¿estarán preparados para responder?

María José Canel, La comunicación de la administración pública. Para gobernar con la sociedad, México, Fondo de Cultura Económica, 2018, 542 pp.

Por Edgar E. Ramírez de la Cruz, CIDE

a publicación del libro La comunicación de la administración pública. Para gobernar con la sociedad es un motivo para celebrar. La obra de la doctora María José Canel es, sin duda, un texto necesario para la enseñanza de esta disciplina. Quienes nos dedicamos a la docencia con frecuencia debemos recurrir a obras estadounidenses que, si bien son útiles, suelen dejar de lado aspectos que hacen única la comunicación de la administración pública (AP) en países como México. Además, es destacable el esfuerzo de la autora por ofrecer una mirada comprensiva sobre el tema, lo que hace el texto útil, no sólo para docentes y estudiantes, sino también para practicantes de la AP.

De manera general, el libro trata una amplia variedad de aspectos relacionados con dos grandes áreas del conocimiento: por un lado, cubre temas relevantes desde las ciencias de la comunicación y, por otro, los enlaza de una forma ágil con los estudios sobre AP. Esto permite que la autora defina un concepto de AP y que, además, identifique las dificultades que el contexto social presenta hoy, tanto para la práctica de la gestión pública en general, como para la aplicación específica del concepto de comunicación propuesto. 